\title{
Facharztprüfung - was gilt? (Stand 2004)
}

Ch. Hänggeli, Geschäftsleiter Sekretariat Aus-, Weiter- und Fortbildung (AWF)

Der Zentralvorstand setzt per 1. Januar 2004 auch die Facharztprüfungen in Kinder- und Jugendmedizin, Plastische, rekonstruktive und ästhetische Chirurgie sowie Prävention und Gesundheitswesen mit eliminatorischer Wirkung in Kraft.

\begin{tabular}{|c|c|c|c|c|c|}
\hline \multicolumn{2}{|c|}{$\begin{array}{l}\text { Tabelle } \\
\text { Muss ich die Facharztprüfung bestehen? } \\
\text { (Fortsetzung auf der folgenden Seite) } \\
\text { Facharzttitel } \\
\text { Inkraftsetzungstermin }\end{array}$} & \multirow[t]{2}{*}{ 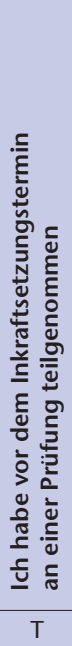 } & 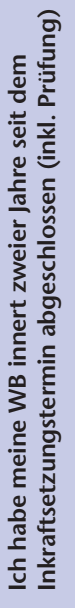 & 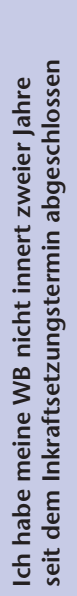 & 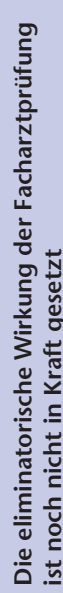 \\
\hline Allergologie und klinische Immunologie & 1.1 .2003 & & $\mathrm{~T}$ & B & - \\
\hline Allgemeinmedizin & 1.1 .2000 & $\mathrm{~T}$ & $T$ & B & - \\
\hline Anästhesiologie & 2.4.1986 & - & - & B & - \\
\hline Angiologie & $1.1 .2005 ?$ & - & - & - & $\mathrm{T}$ \\
\hline Arbeitsmedizin & 1.1 .2001 & $\mathrm{~T}$ & $\mathrm{~T}$ & B & - \\
\hline Chirurgie (inkl. Basisexamen) & 1.1.1999 & $\mathrm{T}$ & $\mathrm{T}$ & B & - \\
\hline Dermatologie und Venerologie & 1.1 .2003 & $\mathrm{~T}$ & $\mathrm{~T}$ & B & - \\
\hline Endokrinologie / Diabetologie & 1.1 .2001 & $\mathrm{~T}$ & $\mathrm{~T}$ & B & - \\
\hline Gastroenterologie & 1.1 .2000 & $\mathrm{~T}$ & $\mathrm{~T}$ & B & - \\
\hline Gynäkologie und Geburtshilfe & 1.1.1999 & $\mathrm{T}$ & $\mathrm{T}$ & B & - \\
\hline Hämatologie & 1.1 .2001 & $\mathrm{~T}$ & $\mathrm{~T}$ & B & - \\
\hline Herz- und thorakale Gefässchirurgie & $1.1 .2005 ?$ & - & - & - & $\mathrm{T}$ \\
\hline Infektiologie & 1.1 .2003 & $\mathrm{~T}$ & $\mathrm{~T}$ & B & - \\
\hline Innere Medizin & 1.1.1999 & $\mathrm{T}$ & $\mathrm{T}$ & B & - \\
\hline Intensivmedizin & 1.1 .2001 & $T$ & $T$ & B & - \\
\hline Kardiologie & 1.1.1999 & $\mathrm{T}$ & $\mathrm{T}$ & B & - \\
\hline Kiefer- und Gesichtschirurgie & $1.1 .2005 ?$ & - & - & - & $\mathrm{T}$ \\
\hline Kinderchirurgie & 2.4 .1986 & - & - & B & - \\
\hline Kinder- und Jugendmedizin & 1.1 .2004 & $\mathrm{~T}$ & $\mathrm{~T}$ & B & - \\
\hline Kinder- und Jugendpsychiatrie und -psychotherapie & 1.1 .2002 & $\mathrm{~T}$ & $\mathrm{~T}$ & B & - \\
\hline Klinische Pharmakologie und Toxikologie & $1.1 .2005 ?$ & - & - & - & $T$ \\
\hline Medizinische Genetik & $1.1 .2005 ?$ & - & - & - & $\mathrm{T}$ \\
\hline Medizinische Onkologie & 1.1 .2001 & $\mathrm{~T}$ & $\mathrm{~T}$ & B & - \\
\hline Nephrologie & $1.1 .2005 ?$ & - & - & - & $\mathrm{T}$ \\
\hline Neurochirurgie & 2.4 .1986 & - & - & B & - \\
\hline Neurologie & 1.1 .2003 & $\mathrm{~T}$ & $\mathrm{~T}$ & B & - \\
\hline Nuklearmedizin & 2.4 .1986 & - & - & B & - \\
\hline Ophthalmologie & 1.1 .2002 & $\mathrm{~T}$ & $\mathrm{~T}$ & B & - \\
\hline ORL (ohne Hals- und Gesichtschirurgie) & 1.1.2001 & $\mathrm{T}$ & $\mathrm{T}$ & B & - \\
\hline Orthopädische Chirurgie & 1.1 .2003 & $T$ & $T$ & B & - \\
\hline
\end{tabular}

Per 1. Januar 2004 hat der Zentralvorstand in drei weiteren Fachgebieten die eliminatorische Wirkung der Facharztprüfung in Kraft gesetzt. Damit wird jetzt für praktisch alle Facharzttitel das Bestehen der Facharztprüfung gefordert, selbstverständlich unter Vorbehalt der anwendbaren Übergangsbestimmungen.

\section{Übergangsbestimmungen}

Ausnahmen gelten für Kandidatinnen und Kandidaten, welche eine der beiden folgenden Bedingungen erfüllen:

- Wer vor dem Inkraftsetzungstermin bereits an einer Facharztprüfung (ganz oder teilweise) teilgenommen hat, muss keine weitere Prüfung absolvieren.

- Wer die Weiterbildung innert zweier Jahre nach dem Inkraftsetzungstermin abgeschlossen hat, benötigt für die Titelerteilung lediglich eine Teilnahmebestätigung über die absolvierte Facharztprüfung. Achtung: Sämtliche Voraussetzungen müssen innert zweier Jahre nach dem Inkraftsetzungstermin erfüllt sein (inkl. komplette Facharztprüfung, ausser wenn die Fachgesellschaft die Zulassung zum 2. Teil wegen eines ungenügenden 1 . Teils verweigert)!

In folgenden Fachgebieten ist weiterhin nur die Teilnahme an der Facharztprüfung gefordert, mit anderen Worten: Das Bestehen der Prüfung ist nicht Voraussetzung für die Erteilung des Facharzttitels.

- Angiologie;

- Herz- und thorakale Gefässchirurgie;

- Kiefer- und Gesichtschirurgie;

- Klinische Pharmakologie und Toxikologie;

- Medizinische Genetik;

- Nephrologie. 


\begin{tabular}{|c|c|c|c|c|c|}
\hline $\begin{array}{l}\text { Tabelle } \\
\text { Muss ich die Facharztprüfung bestehen? } \\
\text { (Fortsetzung von der vorhergehenden Seite) }\end{array}$ & Ingstermin & 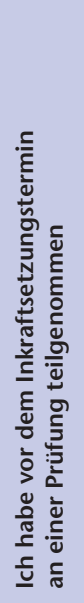 & 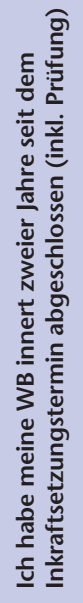 & 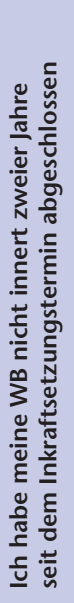 & 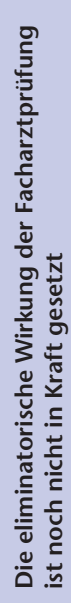 \\
\hline Pathologie & 1.1 .1999 & $\mathrm{~T}$ & $\mathrm{~T}$ & B & - \\
\hline Pharmazeutische Medizin & 1.1 .2003 & $\mathrm{~T}$ & $T$ & B & - \\
\hline Physikalische Medizin und Rehabilitation & 1.1 .2003 & $\mathrm{~T}$ & $\mathrm{~T}$ & B & - \\
\hline Plastische, rekonstruktive und ästhetische Chirurgie & 1.1 .2004 & $\mathrm{~T}$ & $T$ & B & - \\
\hline Pneumologie & 1.1 .2003 & $T$ & $T$ & B & - \\
\hline Prävention und Gesundheitswesen & 1.1 .2004 & $\mathrm{~T}$ & $\mathrm{~T}$ & B & - \\
\hline Psychiatrie und Psychotherapie & 1.1 .2001 & $\mathrm{~T}$ & $\mathrm{~T}$ & B & - \\
\hline Radiologie & 2.4.1986 & - & - & B & - \\
\hline Radio-Onkologie / Strahlentherapie & 2.4.1986 & - & - & B & - \\
\hline Rechtsmedizin & 1.1 .2001 & $T$ & $T$ & B & - \\
\hline Rheumatologie & 1.1 .2001 & $T$ & $T$ & B & - \\
\hline Tropen- und Reisemedizin & 1.1 .2001 & $T$ & $\mathrm{~T}$ & B & - \\
\hline Urologie & 1.1 .2002 & $T$ & $\mathrm{~T}$ & B & - \\
\hline \multicolumn{6}{|l|}{ Schwerpunkte } \\
\hline Neonatologie & 1.1 .2003 & $\mathrm{~T}$ & $\mathrm{~T}$ & B & - \\
\hline Neuropädiatrie & 1.1 .2003 & $T$ & $T$ & B & - \\
\hline Pädiatrische Kardiologie & 1.1 .2001 & $T$ & $T$ & B & - \\
\hline alle anderen Schwerpunkte & & - & - & - & $T$ \\
\hline \multicolumn{6}{|c|}{$\begin{array}{l}\text { B = Bestehen; } T=\text { Teilnehmen. } \\
\text { Die gesamte Weiterbildungsdokumentation (mit den aktuellen Prüfungsterminen) ist auf dem Internet } \\
\text { unter www.fmh.ch/awf abrufbar. Die Prüfungstermine werden auch in der Schweizerischen Ärztezei- } \\
\text { tung publiziert. Bei allfälligen Rückfragen steht Ihnen das Sekretariat Aus-, Weiter- und Fortbildung } \\
\text { (AWF) der FMH gerne zur Verfügung. Adresse: Postfach 293, Elfenstrasse 18, } 3000 \text { Bern 16, Tel. } 031 \\
35911 \text { 11, Fax } 03135911 \text { 12, E-Mail: diplome@hin.ch.a }\end{array}$} \\
\hline
\end{tabular}

